\title{
Eficiência de uso de nitrogênio em cultivares de trigo pioneiras e modernas
}

\author{
Eduardo Beche(1), Giovani Benin(1), Elesandro Bornhofen(1), Samuel Cristian Dalló(1), \\ Luiz Henrique Scarparo Sassi ${ }^{(1)}$ e Ronaldo de Oliveira ${ }^{(1)}$
}

\begin{abstract}
(1)Universidade Tecnológica Federal do Paraná, Rodovia PR 469, Km 01, CEP 85501-970 Pato Branco, PR, Brasil. E-mail: eduardo_beche@hotmail.com, benin@utfpr.edu.br, elesandro.bornhofen@gmail.com, samu_dallo@hotmail.com, luiz_sassi_@hotmail.com, ronaldocampoere@hotmail.com
\end{abstract}

\begin{abstract}
Resumo - O objetivo deste trabalho foi caracterizar cultivares brasileiras de trigo, desenvolvidas em diferentes décadas, quanto à eficiência de uso de $\mathrm{N}$, sob diferentes disponibilidades do nutriente. Dez cultivares de trigo, lançadas entre 1940 e 2009, foram submetidas a quatro doses de adubação nitrogenada $\left(0,60,120\right.$ e $180 \mathrm{~kg} \mathrm{ha}^{-1}$ de N), em ambiente controlado, em delineamento de blocos ao acaso, com três repetições. A produtividade de grãos aumentou em $0,54,0,74,0,74$ e $0,82 \%$ ao ano, nas doses de $0,60,120$ e $180 \mathrm{~kg} \mathrm{ha}^{-1} \mathrm{de} \mathrm{N}$, respectivamente. As eficiências de absorção e de utilização de $\mathrm{N}$ estiveram positivamente associadas à eficiência de uso do nitrogênio, com estreitamento na relação em condições de alta disponibilidade de $\mathrm{N}$. As cultivares modernas são mais eficientes no uso do $\mathrm{N}$ e toleram doses mais baixas do nutriente, em comparação às cultivares pioneiras. Para o desenvolvimento de cultivares mais eficientes no uso de $\mathrm{N}$, os programas de melhoramento genético de trigo devem priorizar a seleção de genótipos com maiores eficiências de absorção, remobilização e utilização de N.
\end{abstract}

Termos para indexação: Triticum aestivum, eficiência de absorção, eficiência de utilização, melhoramento genético, remobilização de nitrogênio, seleção genética.

\section{Nitrogen use efficiency of pioneer and modern wheat cultivars}

\begin{abstract}
The objective of this work was to characterize Brazilian wheat cultivars, developed in different decades, as to their nitrogen use efficiency, under different availabilities of the nutrient. Ten wheat cultivars, released between 1940 and 2009, were subjected to four nitrogen fertilization rates $(0,60,120$, and $180 \mathrm{~kg} \mathrm{ha}^{-1} \mathrm{~N}$ ), under a controlled environment, in a randomized complete block design, with three replicates. Grain yield increased in $0.54,0.74,0.74$, and $0.82 \%$ per year at the rates of $0,60,120$, and $180 \mathrm{~kg} \mathrm{ha}^{-1} \mathrm{~N}$, respectively. Nitrogen uptake and utilization efficiencies were positively associated with $\mathrm{N}$ use efficiency, with strengthening relation under high $\mathrm{N}$ availability conditions. Modern cultivars are more efficient in $\mathrm{N}$ use and tolerate lower rates of the nutrient in comparison to pioneer cultivars. For the development of more efficient cultivars in $\mathrm{N}$ use, wheat-breeding programs should prioritize the selection of genotypes with higher $\mathrm{N}$ uptake, remobilization, and utilization efficiencies.
\end{abstract}

Index terms: Triticum aestivum, uptake efficiency, utilization efficiency, genetic breeding, nitrogen remobilization, genetic selection.

\section{Introdução}

O nitrogênio está entre os nutrientes mais limitantes para a produção agrícola e é o mais utilizado na agricultura mundial. No entanto, apenas 40 a $60 \%$ do $\mathrm{N}$ mineral aplicado é absorvido pela cultura do trigo (Barraclough et al., 2010; Górny et al., 2011), com menor aproveitamento, principalmente, em doses mais elevadas. Assim, a melhoria na eficiência de uso de nitrogênio (EUN) é essencial para o desenvolvimento de uma agricultura sustentável. Essa eficiência consiste da relação entre a produtividade de grãos, ou da parte aérea, e a quantidade de $\mathrm{N}$ disponível no solo ou aplicado (Moll et al., 1982). A EUN pode ser dividida em dois componentes principais: eficiência na absorção de $\mathrm{N}$ (EAN), definida como a capacidade de a planta absorver $\mathrm{N}$; e eficiência na utilização de $\mathrm{N}$ (EUtN), definida como a capacidade de a planta usar o N para produção de grãos (Moll et al., 1982). O comportamento diferencial de genótipos brasileiros de trigo, em diferentes disponibilidades de $\mathrm{N}$, indica que a seleção para eficiência de uso do nutriente pode ser intensificada nos programas de melhoramento genético de trigo (Benin et al., 2012). 
Estratégias para o incremento da EUN têm sido objeto de estudo em todo o mundo (Xu et al., 2012; Cormier et al., 2013; Gaju et al., 2014). É comum o relato de diferenças entre genótipos quanto aos componentes da EUN: EAN, EUtN e índice de colheita de N (ICN) (Foulkes et al., 2009; Barraclough et al., 2010; Górny et al., 2011; Gaju et al., 2011, 2014).

Alguns estudos associam a magnitude da variabilidade em EUtN e EAN, em trigo, à dose de $\mathrm{N}$ aplicada. Com doses baixas, a EAN explica a maior parte da resposta observada em EUN (Ortiz-Monasterio et al., 1997; Foulkes et al., 1998; Le Gouis et al., 2000), enquanto que, em doses altas, destaca-se a importância da EUtN (Brancourt-Hulmel et al., 2003). Entretanto, não há consenso entre os diferentes estudos, pois tanto a EAN quanto a EUtN podem contribuir para o incremento da EUN (Barraclough et al., 2010; Le Gouis et al., 2010; Gaju et al., 2011).

No melhoramento com vistas ao aumento na EUN, o primeiro passo é a definição do nível de disponibilidade de $\mathrm{N}$ no solo (Cormier et al., 2013). Normalmente, as cultivares de trigo são desenvolvidos sob baixa pressão de seleção quanto aos componentes da EUN (Górny et al., 2011). Estudos apontam para a adaptação de cultivares modernas à baixa disponibilidade de $\mathrm{N}$, mesmo que estas tenham sido desenvolvidas em ambientes com elevada disponibilidade do nutriente (Ortiz-Monasterio et al., 1997; Foulkes et al., 1998; Reynolds et al., 1999; Guarda et al., 2004; Górny et al., 2006).

A identificação de caracteres que maximizem a EUN, bem como a caracterização de sua variabilidade, pode contribuir com os programas de melhoramento de trigo na geração de cultivares, com menor exigência pelo N (Gaju et al., 2011). Além disso, há carência desse tipo de informação para cultivares de trigo brasileiras, e não se tem notícia do eventual êxito dos programas de melhoramento genético em incrementar a EUN das cultivares ao longo do tempo.

O objetivo deste trabalho foi caracterizar cultivares brasileiras de trigo, desenvolvidas em diferentes décadas, quanto à eficiência de uso de $\mathrm{N}$, sob diferentes disponibilidades do nutriente.

\section{Material e Métodos}

Dez cultivares de trigo (Triticum aestivum L.), desenvolvidas por diferentes instituições e empresas, disponibilizadas para cultivo no período entre 1940 e 2009, foram avaliadas em casa de vegetação, na safra agrícola de inverno de 2011, em Pato Branco, no Estado do Paraná. As cultivares avaliadas foram divididas em dois grupos, descritos a seguir: grupo de cultivares pioneiras, formado por Frontana (lançada por Iwar Beckman, em 1940), Toropi (por Embrapa Trigo, em 1965) e BR 23 (por Embrapa Trigo, em 1987); e grupo de cultivares modernas, formado por BRS 179 (por Embrapa Trigo, em 1999), BRS 208 (por Embrapa Soja, em 2001), BRS 220 (por Embrapa Soja, em 2003), BRS Guamirim (por Embrapa Trigo, em 2005), BRS Tangará (por Embrapa Soja, em 2007), CD 117 (por Coodetec, em 2008) e Quartzo (por OR Sementes/Biotrigo, em 2009).

O desempenho das cultivares foi avaliado sob diferentes disponibilidades de $\mathrm{N}$, simuladas em quatro níveis de adubação: 0 e $60 \mathrm{~kg} \mathrm{ha}^{-1} \mathrm{de} \mathrm{N}$, que representam baixa disponibilidade do nutriente; e 120 e $180 \mathrm{~kg} \mathrm{ha}^{-1}$ de $\mathrm{N}$, que representam alta disponibilidade. A aplicação de $\mathrm{N}$ foi feita em três etapas: primeira etapa, $30 \mathrm{~kg} \mathrm{ha}^{-1}$ na base, para todos os tratamentos, exceto o controle; segunda etapa, $65 \%$ do restante de $\mathrm{N}$ no início do perfilhamento ( $Z$ 22, na escala Zadoks); e terceira etapa, $35 \%$ da dose no final do perfilhamento ( $Z$ 39). A ureia diluída em água ( $45 \%$ de $N$ ) foi utilizada como fonte de $\mathrm{N}$. As fertilizações de $\mathrm{N}$ corresponderam aos seguintes parcelamentos, nas três etapas: $0 \mathrm{~kg} \mathrm{ha}^{-1}, 0+0+0$; $60 \mathrm{~kg} \mathrm{ha}^{-1}, 30+19,5+10,5 ; 120 \mathrm{~kg} \mathrm{ha}^{-1}, 30+58,5+31,5$; $180 \mathrm{~kg} \mathrm{ha}^{-1}, 30+97,5+52,5$. O experimento foi disposto em delineamento de blocos ao acaso, em arranjo fatorial simples $4 \times 10$ (quatro doses de $\mathrm{N}$ e dez cultivares de trigo), com três repetições. Cada unidade experimental foi composta por dois vasos de $20 \mathrm{~L}$, que receberam 25 sementes pré-germinadas (papel de filtro, $22^{\circ} \mathrm{C}$, por 24 horas) cada um, o que representou uma densidade de semeadura de 300 sementes por metro quadrado.

O solo utilizado nos vasos apresentava as seguintes características químicas: $\mathrm{pH}$ em $\mathrm{CaCl}_{2}$ de 4,77; $8,34 \mathrm{cmol}_{\mathrm{c}} \mathrm{dm}^{-3}$ de $\mathrm{H}+\mathrm{Al} ; 0,13 \mathrm{cmol}_{\mathrm{c}} \mathrm{dm}^{-3}$ de $\mathrm{Al}^{+3}$; 4,73 $\mathrm{cmol}_{\mathrm{c}} \mathrm{dm}^{-3}$ de $\mathrm{Ca}^{+2} ; 2,64 \mathrm{cmol}_{\mathrm{c}} \mathrm{dm}^{-3}$ de $\mathrm{Mg}^{2+}$; $0,34 \mathrm{cmol}_{\mathrm{c}} \mathrm{dm}^{-3} \mathrm{de} \mathrm{K}^{+} ; 2,11 \mathrm{mg} \mathrm{dm}{ }^{-3}$ de P (Mehlich); $58,6 \mathrm{~g} \mathrm{dm}^{-3}$ de matéria orgânica; saturação por bases de $48,1 \%$; e saturação por alumínio de $1,66 \%$. O pH do solo foi corrigido com calcário dolomítico (PRNT de 87\%), na dose de $3 \mathrm{Mg} \mathrm{ha}^{-1}$, tendo-se buscado um valor de saturação de bases de $70 \%$. Da mesma forma, 
o solo foi corrigido com potássio e fósforo, na dose de $60 \mathrm{~kg} \mathrm{ha}^{-1}$.

$\mathrm{Na}$ antese (Z 60), foram coletadas cinco plantas por unidade experimental, para determinar a quantidade de $\mathrm{N}$ acumulado na parte aérea. As plantas foram colhidas, em cada vaso, no estádio de maturação fisiológica (Z 90). As frações de grãos e palha (parte aérea das plantas) foram separadas manualmente; os grãos foram pesados e a sua umidade foi padronizada a $13 \%$, para determinação da produtividade de grãos por vaso. A palha foi seca em estufa, a $40^{\circ} \mathrm{C}$, por 48 horas, e triturada em moinho de facas. Em seguida, foram retiradas amostras de palha e grãos, que foram submetidas à análise química para determinação da concentração de $\mathrm{N}$, de acordo com o método de Kjeldahl (Tedesco et al., 1995).

$\mathrm{O}$ acúmulo de $\mathrm{N}$ total nos grãos e na parte aérea, durante a antese e na maturidade fisiológica, foi determinado pela multiplicação do teor de $\mathrm{N}$ em cada fração pela produção de grãos e de biomassa, respectivamente. Os componentes da eficiência de uso foram calculados conforme Moll et al. (1982): EUN $\left(\mathrm{g} \mathrm{g}^{-1}\right)=$ PG/SN, em que PG é a produção de grãos (g) e SN é a quantidade de $\mathrm{N}$ fornecida pelo solo + fertilizantes $(\mathrm{g})$, tendo-se determinado o nitrogênio fornecido pelo solo a partir do conteúdo total de nitrogênio presente na amostra de solo (Tedesco et al., 1995); EAN $\left(\mathrm{g} \mathrm{g}^{-1}\right)=(\mathrm{NG}+\mathrm{NP}) / \mathrm{SN}$, em que NG é a quantidade de $\mathrm{N}$ nos grãos ( $\mathrm{g}$ ) e NP é a quantidade de $\mathrm{N}$ na palha (g), na maturação fisiológica; EUtN $\left(\mathrm{g} \mathrm{g}^{-1}\right)=\mathrm{PG} /(\mathrm{NG}+\mathrm{NP})$; índice de colheita do $\mathrm{N}, \mathrm{ICN}(\%)=\mathrm{CNG} \times \mathrm{PG} /(\mathrm{CNG}+\mathrm{CNP}) \times \mathrm{BIO}, \mathrm{em}$ que $\mathrm{CNG}$ é a concentração de $\mathrm{N}$ nos grãos (\%), CNP é a concentração na palha (\%) e BIO é a biomassa total (palha+grãos) (g); fração aparente de recuperação de $\mathrm{N}, \operatorname{FARN}(\%)=\left(\mathrm{EAN}_{\mathrm{Nx}}-\mathrm{EAN}_{\mathrm{N} 0}\right) / \mathrm{NX}$, em que $\mathrm{EAN}_{\mathrm{Nx}}$ é a eficiência de absorção na dose maior de $\mathrm{N}, \mathrm{EAN}_{\mathrm{N} 0}$ é a eficiência de absorção na dose menor e NX é a dose maior de $\mathrm{N}(\mathrm{g})$; eficiência de remobilização de $\mathrm{N}$, ERN $(\%)=$ NR/NTA, em que NTA é o N total na antese (g) e NR é o N remobilizado da biomassa para os grãos (g), obtido pela subtração da quantidade de $\mathrm{N}$ na palha durante a maturação pela quantidade de $\mathrm{N}$ na biomassa total da parte aérea durante a antese (NBA, g); absorção de $\mathrm{N}$ pós-antese, ANPA (g) = NBM - NBA, em que NBM é a quantidade de $\mathrm{N}$ na biomassa total da parte aérea durante a maturação $(\mathrm{g})$; índice de resposta à EUtN, $I_{\mathrm{EUtN}}=\left(\mathrm{PG}_{\mathrm{Nx}}-\mathrm{PG}_{\mathrm{N} 0}\right) /\left(\mathrm{NA}_{\mathrm{Nx}}-\mathrm{NA} \mathrm{A}_{\mathrm{N} 0}\right)$, em que
$\mathrm{PG}_{\mathrm{Nx}}$ é a produção de grãos na dose maior de $\mathrm{N}(\mathrm{g})$, $\mathrm{PG}_{\mathrm{N} 0}$ é produção na dose menor $(\mathrm{g}), \mathrm{NA}_{\mathrm{Nx}}$ é quantidade de $\mathrm{N}$ absorvido na dose maior de $\mathrm{N}(\mathrm{g})$ e $\mathrm{NA}_{\mathrm{N} 0}$ é a quantidade de $\mathrm{N}$ absorvido na dose menor (g); e índice de tolerância à baixa disponibilidade de $\mathrm{N}, \mathrm{T}=\mathrm{PG}_{\mathrm{N} 0} /$ $\mathrm{PG}_{\mathrm{N} x} / \mathrm{D}$, em que $\mathrm{D}$ é obtido da divisão da média geral da produtividade de grãos na dose menor de $\mathrm{N}(\mathrm{g})$ pela média da produtividade na dose maior (g) (Górny et al., 2011).

Procedeu-se à análise de variância univariada no modelo fatorial simples. As médias dos tratamentos foram comparadas pelo teste de Tukey, a 5\% de probabilidade. A comparação entre os anos de lançamento das cultivares foi realizada mediante regressão polinomial. Procedeu-se à análise de correlação simples de Pearson entre os caracteres avaliados.

Além disso, a identificação dos efeitos diretos e indiretos dos caracteres sobre a PG foi feita por meio da análise de trilha (Wright, 1921), após o teste e a identificação de baixa multicolinearidade entre as variáveis, por número de condição $(\mathrm{NC})$ menor que 100 (Montgomery \& Peck, 1981). A análise de trilha foi feita para os caracteres PG, EAN, ERN, EUtN, ICN e ANPA, em duas condições de disponibilidade de N: baixa, com doses de 0 e $60 \mathrm{~kg} \mathrm{ha}^{-1}$ de $\mathrm{N}$; e alta, com doses de 120 e $180 \mathrm{~kg} \mathrm{ha}^{-1}$ de $\mathrm{N}$.

As análises foram realizadas com o programa computacional Genes (Cruz, 2013), e as figuras foram elaboradas com o programa SigmaPlot, versão 11 (Systat Software, San Jose, CA, EUA). A acurácia seletiva foi calculada pela expressão: $\mathrm{AS}=(1-1 / \mathrm{F})^{0,5}$, em que $F$ (de Snedecor) é o valor da razão entre variâncias, associado aos efeitos de tratamentos (cultivares) (Resende \& Duarte, 2007).

\section{Resultados e Discussão}

A interação entre as cultivares de trigo e as doses de nitrogênio foi significativa para todos os caracteres avaliados $(p \leq 0,01)$. As acurácias seletivas dos caracteres avaliados oscilaram entre 0,87 e 0,98 , e indicaram boa precisão experimental (Resende \& Duarte, 2007). De modo similar, as amplitudes dos coeficientes de variação $(5,9$ a $15,2 \%)$ também foram de baixa magnitude. A produtividade foi maior nas cultivares modernas, em todas as doses de $\mathrm{N}$ avaliadas (Figura 1), e variaram de $19 \mathrm{~g}$ por vaso, na dose de 
$0 \mathrm{~kg} \mathrm{ha}^{-1}$ de $\mathrm{N}$, a $52 \mathrm{~g}$ por vaso, na dose de $180 \mathrm{~kg} \mathrm{ha}^{-1}$ de N. Os ganhos em produtividade no período de 1940 a 2009 foram de $0,54,0,74,0,74$ e $0,82 \%$ ao ano, nas doses de 0,60, 120 e $180 \mathrm{~kg} \mathrm{ha}^{-1}$ de N, respectivamente. Esses resultados estão de acordo com os obtidos em outros estudos (Brancourt-Hulmel et al., 2003; Guarda et al., 2004). Cormier et al. (2013) relataram ganho genético de $0,45 \%$ ao ano para produtividade, independentemente da disponibilidade de $\mathrm{N}$.

As cultivares modernas apresentaram maior EUN que as pioneiras, em todas as doses de N (Figura 2). As respostas da EUN à adubação nitrogenada variaram expressivamente (amplitude de 10 a 75 g de grão por grama de $\mathrm{N}$ ), o que corrobora os resultados de Sylvester-Bradley \& Kindred (2009). A EUN diminuiu com a elevação das doses de $\mathrm{N}$, provavelmente em razão do aumento gradual na produtividade de grãos com a adubação nitrogenada (Zhao et al., 2006; Rahimizadeh et al., 2010; Cormier et al., 2013) e da baixa eficiência de uso do nutriente, em torno de 40 a 60\% (Barraclough et al., 2010; Górny et al., 2011). Os maiores valores de EUN foram obtidos nas doses de 0 e $60 \mathrm{~kg} \mathrm{ha}^{-1}$ de $\mathrm{N}$, independentemente da cultivar. Na média das doses, obteve-se um progresso de $0,59 \%$ por ano $\left(\mathrm{R}^{2}=0,73\right)$ para a EUN. Cormier et al. (2013) constataram ganho de $0,33 \%$ ao ano, principalmente em razão do aumento na EUtN.

A EAN aumentou linearmente no período de 1940 a 2009, em todas as doses de $\mathrm{N}$ testadas $\left(0 \mathrm{~kg} \mathrm{ha}^{-1}\right.$ de N, R ${ }^{2}=0,82 ; 60 \mathrm{~kg} \mathrm{ha}^{-1}$ de $\mathrm{N}, \mathrm{R}^{2}=0,81 ; 120 \mathrm{~kg} \mathrm{ha}^{-1}$ de $\mathrm{N}, \mathrm{R}^{2}=0,84$; e $180 \mathrm{~kg} \mathrm{ha}^{-1}$ de $\mathrm{N}, \mathrm{R}^{2}=0,88$ ), e foi maior nas doses de 180,120 e $60 \mathrm{~kg} \mathrm{ha}^{-1}$ de $\mathrm{N}$ (Figura 2), o que está de acordo com os dados de Barraclough et al. (2010) e Guarda et al. (2004). Portanto, no período de 1940 a 2009, as cultivares aumentaram sua capacidade de absorver e armazenar $\mathrm{N}$ proveniente do solo, o que também foi relatado em outros estudos (Sylvester-Bradley \& Kindred, 2009; Sadras \& Lawson, 2013). O aumento linear da absorção de N pós-antese, no período de 1940 a 2009, independentemente das doses avaliadas, mostra que os programas de melhoramento genético do trigo no Brasil têm contribuído para maximizar a capacidade de a planta absorver $\mathrm{N}$ do solo.

A EUtN também aumentou linearmente no período de 1940 a 2009, em todas as doses de N, o que confirma os resultados de Guarda et al. (2004) e Górny et al. (2006). A EUtN alcançou valor máximo na dose de $0 \mathrm{~kg} \mathrm{ha}^{-1} \mathrm{de} \mathrm{N}\left(27\right.$ a $\left.42 \mathrm{~g} \mathrm{~g}^{-1}\right)$ e menores valores nas doses de 60,120 e $180 \mathrm{~kg} \mathrm{ha}^{-1}$ de N (16 a $30 \mathrm{~g} \mathrm{~g}^{-1}$ ) (Figura 2).
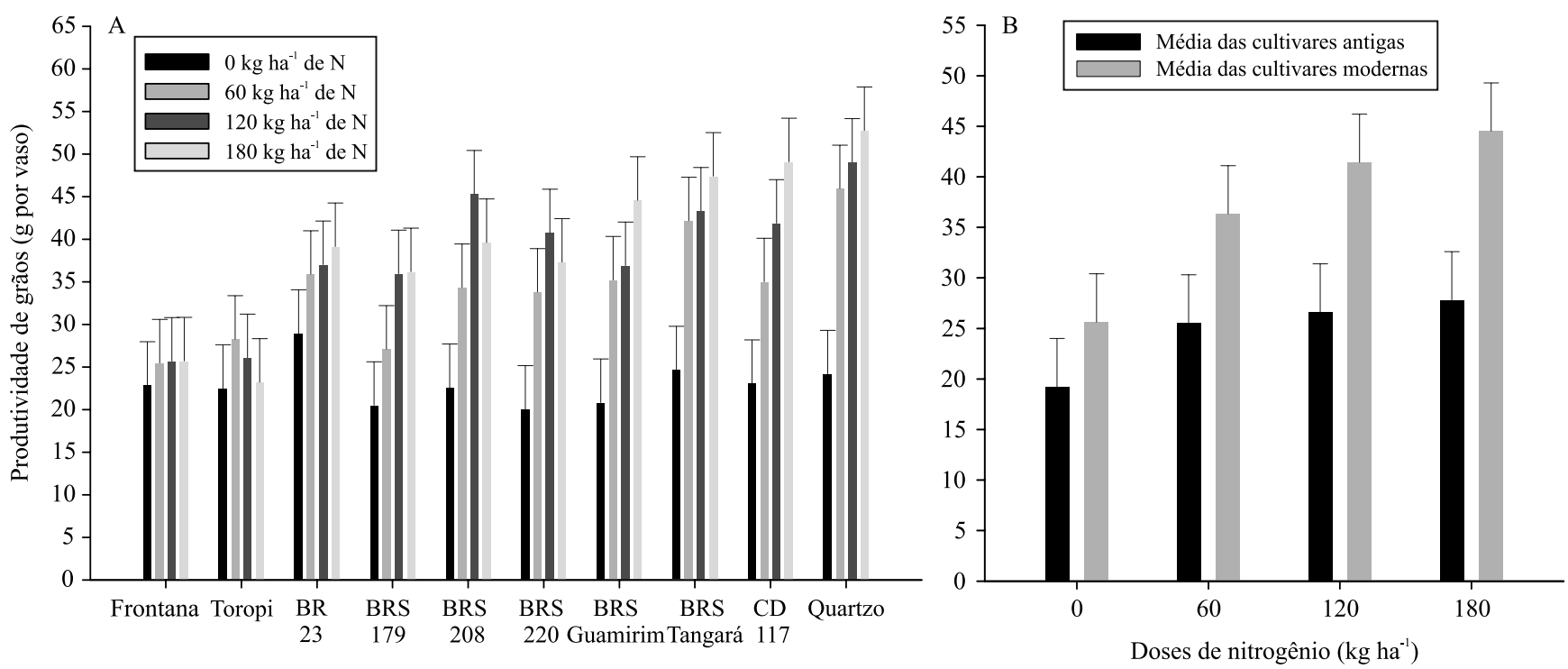

Figura 1. Produtividade de grãos de dez cultivares de trigo (Triticum aestivum) lançadas entre 1940 e 2009 (A), e produtividade média de grãos de cultivares antigas e modernas (B), avaliadas sob quatro doses de N. As barras no topo das colunas representam a DMS-Tukey, a 5\% de probabilidade. 

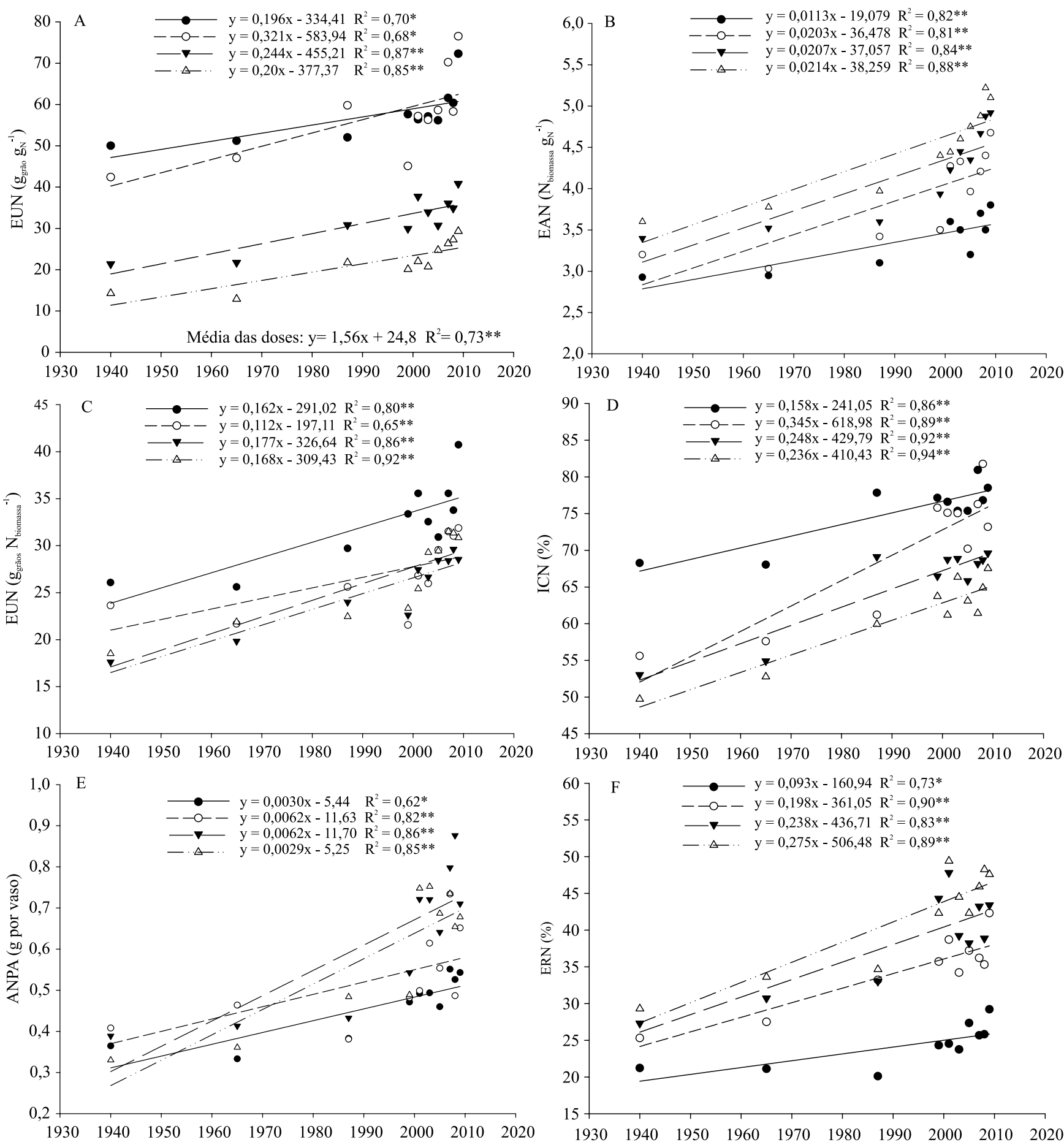

Ano de lançamento

Ano de lançamento

$\multimap 0 \mathrm{~kg} \mathrm{ha}^{-1} \mathrm{deN}-\mathrm{O}_{-6} \mathrm{~kg} \mathrm{ha}^{-1} \mathrm{deN} \longrightarrow-120 \mathrm{~kg} \mathrm{ha}^{-1} \mathrm{deN} \quad \cdots \Delta .180 \mathrm{~kg} \mathrm{ha}^{-1} \mathrm{deN}$

Figura 2. Componentes da eficiência de uso do nitrogênio (EUN, A): eficiência de absorção (EAN, B); eficiência de utilização (EUtN, C); índice de colheita (ICN, D); absorção de N pós-antese (ANPA, E); e eficiência de remobilização de N (ERN, F) de dez cultivares de trigo (Triticum aestivum) lançadas entre 1940 e $2009 .{ }^{*}$ e ${ }^{* *}$ Significativo pelo teste $\mathrm{t}$ a 5 e $1 \%$ de probabilidade, respectivamente. 
Esses resultados são indicativos de que os genótipos não responderam eficientemente ao $\mathrm{N}$ aplicado, quando este apresentava maior disponibilidade (Rahimizadeh et al., 2010). Contudo, mesmo com a baixa resposta da EUtN à adubação nitrogenada, as cultivares modernas foram mais eficientes em utilizar o $\mathrm{N}$ do que as pioneiras. Quando em alta disponibilidade, a EUtN apresentou efeito direto relativamente alto sobre a produtividade de grãos, além de ter contribuído indiretamente para esse caráter, por meio de sua interferência em ANPA e EAN (Tabela 1).

$\mathrm{O}$ efeito de genótipo captou a maior fração da variação em EUtN, o que também foi verificado por Cormier et al. (2013). Esse resultado deve-se ao aumento significativo $(\mathrm{p} \leq 0,01)$ no índice de colheita de N (Figura 2), ao longo dos anos e em todas as doses de $\mathrm{N}$ avaliadas (Brancourt-Hulmel et al., 2003). Os valores de ICN estiveram entre 50 e $80 \%$, variação semelhante à observada por Barraclough et al. (2010) e Gaju et al. (2011).

A ERN também aumentou no período de 1940 a 2009, independentemente da dose de N (Figura 2).
Os valores de ERN variaram de $20 \%$, em baixa oferta de N, a $45 \%$, em alta oferta. Esse caractere apresentou efeito direto sobre a produtividade de grãos $(0,55)$ e contribuiu indiretamente para a produtividade via EAN $(0,47)$ e ANPA $(0,34)$ (Tabela 1$)$. Em geral, observouse que, em baixa oferta de N, ERN e EAN parecem estar mais associadas à produtividade de grãos, ao passo que, em alta oferta de N, EUtN apresenta efeitos diretos superiores, juntamente com EAN. Assim, a seleção para genótipos mais hábeis na absorção de $\mathrm{N}$ pode contribuir, de maneira mais significativa, para o incremento na produtividade.

Os valores de ANPA variaram de 0,35 a $0,5 \mathrm{~g}$ por vaso, nas doses de 0 e $60 \mathrm{~kg} \mathrm{ha}^{-1}$ de $\mathrm{N}$, e de 0,3 a $0,9 \mathrm{~g}$ por vaso, nas doses de 120 e $180 \mathrm{~kg} \mathrm{ha}^{-1}$ de $\mathrm{N}$, o que está de acordo com os resultados obtidos por Gaju et al. (2011), que obtiveram maiores valores de ANPA em altas doses de N. Gaju et al. (2014) relataram que a variação genética no rendimento de grãos parece ser influenciada pelo acúmulo de $\mathrm{N}$ na pré-antese, ao invés do acúmulo na pós-antese. Os autores sugeriram a seleção para ERN com o objetivo de maximizar a produtividade de

Tabela 1. Partição dos coeficientes de correlação de Pearson (r) em efeitos diretos e indiretos, por meio da análise de trilha, entre cinco caracteres componentes da eficiência de uso de nitrogênio (EUN) em condições de baixa e alta disponibilidade de N.

\begin{tabular}{|c|c|c|c|c|c|c|c|}
\hline \multirow[t]{2}{*}{ Componentes da EUN } & \multirow[t]{2}{*}{ Efeitos diretos } & \multicolumn{5}{|c|}{ Efeitos indiretos via } & \multirow[t]{2}{*}{$\mathrm{R}^{2}$} \\
\hline & & EAN & EUtN & $\mathrm{ICN}$ & ERN & ANPA & \\
\hline & \multicolumn{7}{|c|}{ Baixa disponibilidade de $\mathrm{N}$} \\
\hline Eficiência de absorção (EAN) & 0,36 & - & 0,03 & $-0,14$ & 0,47 & 0,10 & $0,82 * *$ \\
\hline Eficiência de utilização (EUtN) & 0,11 & 0,09 & - & $-0,19$ & $-0,03$ & 0,05 & 0,04 \\
\hline Índice de colheita (ICN) & $-0,30$ & 0,16 & 0,07 & - & 0,06 & 0,06 & 0,05 \\
\hline Eficiência de recuperação (ERN) & 0,55 & 0,30 & $-0,01$ & $-0,04$ & - & 0,08 & $0,89 * *$ \\
\hline Eficiência de remobilização (ANPA) & 0,13 & 0,28 & 0,04 & $-0,14$ & 0,34 & - & $0,65^{* *}$ \\
\hline $\mathrm{R}^{2}$ & & & & 0,85 & & & \\
\hline \multirow[t]{2}{*}{ Efeito da variável residual } & & & & 0,38 & & & \\
\hline & \multicolumn{7}{|c|}{ Alta disponibilidade de $\mathrm{N}$} \\
\hline Eficiência de absorção (EAN) & 0,32 & - & 0,37 & 0,16 & 0,19 & $-0,19$ & $0,84 * *$ \\
\hline Eficiência de utilização (EUtN) & 0,40 & 0,29 & - & 0,18 & 0,18 & $-0,19$ & $0,85^{* *}$ \\
\hline Índice de colheita (ICN) & 0,26 & 0,20 & 0,28 & - & 0,15 & $-0,17$ & $0,73^{* *}$ \\
\hline Eficiência de recuperação (ERN) & 0,24 & 0,25 & 0,29 & 0,17 & - & $-0,16$ & $0,79 * *$ \\
\hline Eficiência de remobilização (ANPA) & $-0,23$ & 0,27 & 0,34 & 0,19 & 0,17 & - & $0,74 * *$ \\
\hline $\mathrm{R}^{2}$ & \multicolumn{7}{|c|}{0,81} \\
\hline Efeito da variável residual & \multicolumn{7}{|c|}{0,43} \\
\hline
\end{tabular}

${ }^{{ }^{n}}$ Não significativo. * e **Significativo pelo teste t a 5 e $1 \%$ de probabilidade, respectivamente. 
grãos e a EUN. Em doses intermediárias de N, houve progresso genético de aproximadamente $0,43 \%$ ao ano $\left(\mathrm{R}^{2}=0,66\right)$ para a ERN. Esses resultados evidenciam a necessidade de cautela com a adubação nitrogenada excessiva na cultura do trigo, para que não ocorra consumo de luxo do nutriente (Foulkes et al., 1998; Gaju et al., 2011).

A EAN remete à capacidade de as raízes da planta adquirirem $\mathrm{N}$ do solo, enquanto a EUtN indica a fração do $\mathrm{N}$ adquirido que é convertido em biomassa vegetal ou em grãos. A EUN decorre da integração entre EAN e EUtN, e é controlada por muitos genes (Hirel et al., 2001; Habash et al., 2007; Masclaux-Daubresse et al., 2010), com forte influência do ambiente, representada, no presente trabalho, pela interação entre genótipos e a dose de $\mathrm{N}$ (Xu et al., 2012). A associação entre EUN e seus componentes EAN e EUtN (Figura 3) mostrou que a variação genética encontrada para EUN está mais estreitamente associada à EUtN (baixo $\mathrm{N}$, $\mathrm{r}=0,86$; alto $\mathrm{N}, \mathrm{r}=0,89$ ) do que à $\mathrm{EAN}$ (baixo $\mathrm{N}, \mathrm{r}=0,63$; alto $\mathrm{N}, \mathrm{r}=0,84)$. Esses resultados se assemelham aos de Barraclough et al. (2010) e Gaju et al. (2011). $\mathrm{O}$ aumento da EAN pode diminuir as perdas de $\mathrm{N}$ no solo, ao passo que o aumento da EUtN pode diminuir a concentração de N na palha (Xu et al., 2012).

A FARN aumentou significativamente com o passar dos anos, independentemente da dose de $\mathrm{N}$, e variou de 4 a $15 \%$, na dose de $180 \mathrm{~kg} \mathrm{ha}^{-1}$ de $\mathrm{N}$; de 7 a $45 \%$, na dose de $120 \mathrm{~kg} \mathrm{ha}^{-1}$ de N; e de 24 a $60 \%$, na dose de $60 \mathrm{~kg} \mathrm{ha}^{-1}$ de N (Figura 3). Guarda et al. (2004) encontraram valores de FARN de 14 a 56\%, em baixa disponibilidade de $\mathrm{N}\left(80 \mathrm{~kg} \mathrm{ha}^{-1} \mathrm{de} \mathrm{N}\right)$, e de 8 a 34\%, em alta disponibilidade (160 kg ha-1 de N). Quanto maior a dose de $\mathrm{N}$ utilizada, menor é a sua recuperação, principalmente em razão de perdas por lixiviação (Riley et al., 2001), volatilização (Ma et al., 2010) e baixa EAN (Figura 2).

As cultivares modernas, quando comparadas às pioneiras, apresentaram FARN superior em $24 \%$, na dose de $60 \mathrm{~kg} \mathrm{ha}^{-1}$ de $\mathrm{N}$; em $30 \%$, na de $120 \mathrm{~kg} \mathrm{ha}^{-1}$ de N; e em 10\%, na de $180 \mathrm{~kg} \mathrm{ha}^{-1}$ de N (Figura 3). As alterações em FARN sugerem mudanças na capacidade de absorção de $\mathrm{N}$ pelas plantas, possivelmente ligadas à capacidade de absorção da raiz (King et al., 2003; Foulkes et al., 2004) e à tendência de cultivares modernas apresentarem maior período de perfilhamento, o que aumenta o período de absorção de N (Foulkes et al., 1998).
$\mathrm{O} \mathrm{IR}_{\mathrm{EUtN}}$, que representa a habilidade fisiológica das plantas em responderem a cada unidade adicional de N absorvido (Fageria \& Baligar, 1993), é um importante componente para adaptação das plantas a ambientes com baixa disponibilidade do nutriente (Górny et al., 2011). As cultivares foram agrupadas em quatro quadrantes (grupos) de resposta, de acordo com seus $\mathrm{IR}_{\mathrm{EUtN}}$, em função da produtividade de grãos sob baixa disponibilidade de N (Figura 3). As cultivares modernas CD 117, BRS Guamirim, BRS 208, Quartzo e BRS Tangará enquadraram-se como eficientes e responsivas (quadrante 1), ou seja, apresentaram produtividade superior em baixa dose de $\mathrm{N}$ e foram capazes de responder eficientemente ao incremento de N disponível. As cultivares BRS 220, BRS 179 e BR 23 enquadraram-se como não eficientes, mas responsivas (quadrante 4), ou seja, apresentaram alta produtividade de grãos em baixa disponibilidade de $\mathrm{N}$ e reduzida capacidade de utilizar eficientemente cada unidade adicional de $\mathrm{N}$ disponibilizada. Já as cultivares pioneiras Frontana e Toropi enquadraram-se como não eficientes e não responsivas (quadrante 3). Em termos práticos, as cultivares responsivas à aplicação de $\mathrm{N}$ e com produtividade superior sob baixa disponibilidade de $\mathrm{N}$ (quadrantes 1 e 2) são ideais para programas de melhoramento que visem a adaptação a condições de baixa adubação nitrogenada (Fageria \& Baligar, 1997).

A plotagem do $\mathrm{IR}_{\mathrm{EUtN}}$ em função do índice de tolerância à baixa disponibilidade de $\mathrm{N}$ (Figura 3) revelou que as cultivares modernas Quartzo, BRS Tangará, BRS 220 e BRS 208 foram tolerantes à baixa disponibilidade de $\mathrm{N}$ e eficientes em sua utilização (quadrante 1). As cultivares BRS Guamirim, CD 117, BRS 179 e BR 23 foram responsivas à adubação nitrogenada, mas não toleraram baixa disponibilidade do nutriente (quadrante 4). As cultivares antigas, Frontana e Toropi (quadrante 2), mostraramse tolerantes à baixa disponibilidade de $\mathrm{N}$, mas não foram eficientes em utilizar o nutriente adicionado pela adubação. Em síntese, constatou-se que, com o passar dos anos, o melhoramento aumentou a eficiência de uso de $\mathrm{N}$ e a tolerância das plantas a baixas doses do nutriente.

A caracterização de cultivares quanto à EUN é de grande importância para o uso racional do N. O presente trabalho indica que genótipos com maior eficiência de absorção e utilização de $\mathrm{N}$ apresentam maior EUN, e que a EUtN é mais importante para o conjunto de genótipos avaliados. Estudos que busquem elucidar 

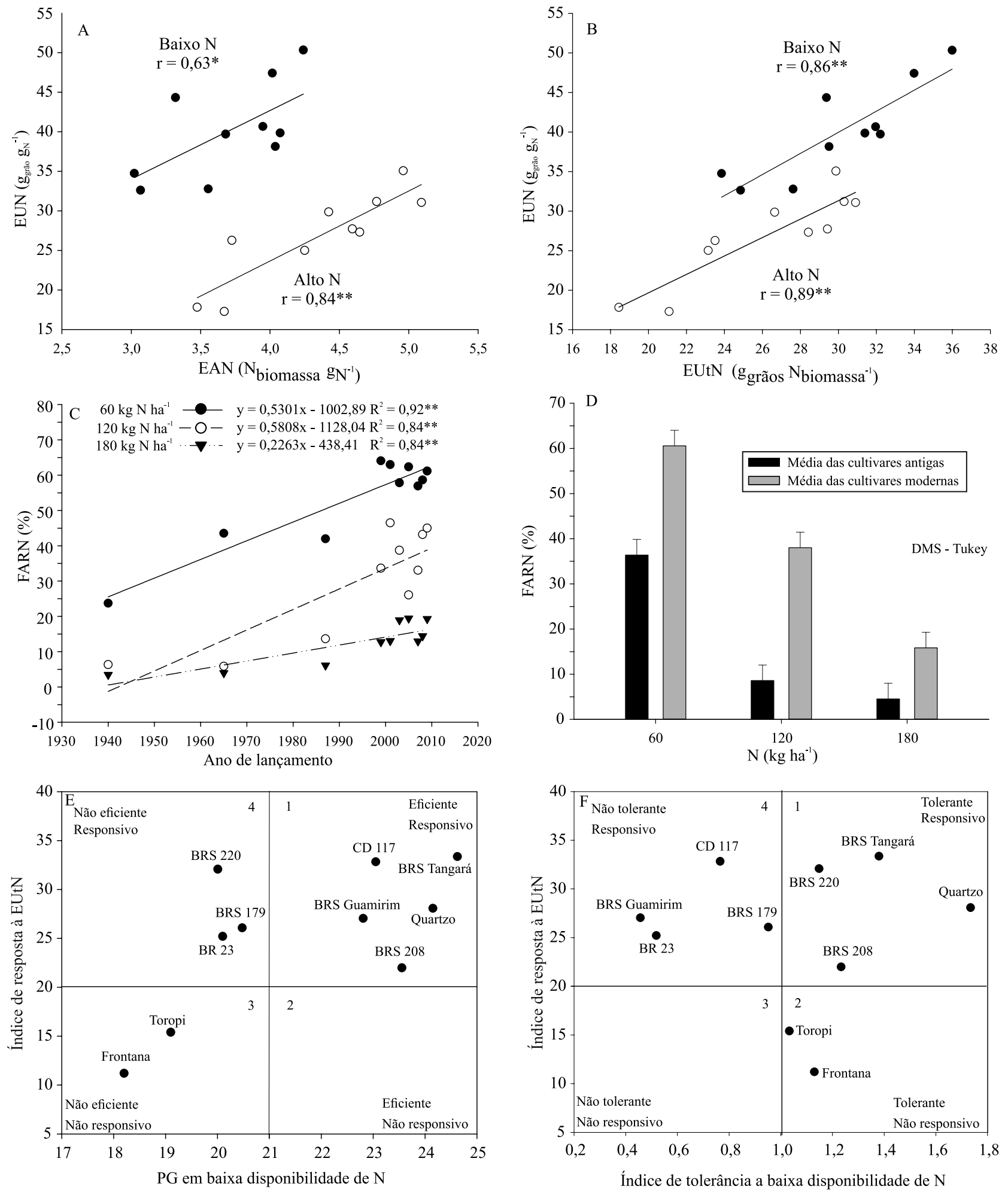

Figura 3. Associação entre eficiência de uso (EUN) e de absorção de $N$ (EAN) sob baixas (0 e $60 \mathrm{~kg} \mathrm{ha}^{-1}$ de N) e altas (120 e $180 \mathrm{~kg} \mathrm{ha}^{-1}$ de N) disponibilidades do nutriente (A); associação entre EUN e eficiência de utilização de N (EUtN), sob baixas e altas disponibilidades do nutriente (B); fração aparente de recuperação de N (FARN, C e D); índices de resposta de cultivares pioneiras e modernas à EUtN em função da produtividade de grãos $(\mathrm{PG})$ sob baixa disponibilidade de $\mathrm{N}(\mathrm{E})$ e da tolerância à baixa disponibilidade do nutriente (F). ns Não significativo. ${ }^{*} \mathrm{e} *$ Significativo pelo teste t a 5 e $1 \%$ de probabilidade, respectivamente. 
as bases genéticas e fisiológicas da translocação, da remobilização e da ciclagem do nutriente nas plantas são importantes para que se possa continuar a promover a eficiência de uso de nitrogênio em trigo.

\section{Conclusões}

1. As eficiências de absorção e de utilização de nitrogênio estão positivamente associadas à eficiência de uso de nitrogênio, independentemente do nível de disponibilidade do nutriente.

2. As cultivares modernas Quartzo, CD 117, BRS Tangará, BRS 220, BRS 208 e BRS Guamirim, lançadas após 2001, são eficientes no uso de $\mathrm{N}$ e mais tolerantes a ambientes com baixa disponibilidade do nutriente, em comparação às cultivares pioneiras.

3. Em condições de baixa oferta de nitrogênio, a eficiência de absorção e de remobilização estão direta e indiretamente associadas à produtividade de grãos; ao passo que, sob alta oferta, a eficiência de absorção e de utilização são mais importantes.

4. Para o desenvolvimento de cultivares mais eficientes no uso de $\mathrm{N}$, os programas de melhoramento genético de trigo devem priorizar a seleção de genótipos com maiores eficiências de absorção, remobilização e utilização de $\mathrm{N}$.

\section{Referências}

BARRACLOUGH, P.B.; HOWARTH, J.R.; JONES, J.; LOPEZ-BELLIDO, R.; PARMAR, S.; SHEPHERD, C.E.; HAWKESFORD, M.J. Nitrogen efficiency of wheat: genotypic and environmental variation and prospects for improvement. European Journal of Agronomy, v.33, p.1-11, 2010. DOI: 10.1016/j.eja.2010.01.005.

BENIN, G.; BORNHOFEN, E.; BECHE, E.; PAGLIOSA, E.S.; SILVA, C.L. da; PINNOW, C. Agronomic performance of wheat cultivars in response to nitrogen fertilization levels. Acta Scientiarum. Agronomy, v.34, p.275-283, 2012. DOI: 10.4025/ actasciagron.v34i3.14468.

BRANCOURT-HULMEL, M.; DOUSSINAULT, G.; LECOMTE, C.; BÉRARD, P.; LE BUANEC, V.; TROTTET, M. Genetic improvement of agronomic traits of winter wheat cultivars released in France from 1946 to 1992. Crop Science, v.43, p.37-45, 2003. DOI: $10.2135 /$ cropsci2003.3700.

CORMIER, F.; FAURE, S.; DUBREUIL, P.; HEUMEZ, E.; BEAUCHÊNE, K.; LAFARGE, S.; PRAUD, A.; LE GOUIS, J. A multi-environmental study of recent breeding progress on nitrogen use efficiency in wheat (Triticum aestivum L.). Theoretical and Applied Genetics, v.126, p.3035-3048, 2013. DOI: 10.1007/ s00122-013-2191-9.
CRUZ, C.D. GENES - a software package for analysis in experimental statistics and quantitative genetics. Acta Scientiarum. Agronomy, v.35, p.271-276, 2013. DOI: 10.4025/ actasciagron.v35i3.21251.

FAGERIA, N.K.; BALIGAR, V.C. Screening crop genotypes for mineral stresses. In: WORKSHOP ON ADAPTATION OF PLANTS TO SOIL STRESSES, 1993, Lincoln. Proceedings. Lincoln: INSTOR MIL, 1993. p.142-159.

FAGERIA, N.K.; BALIGAR, V.C. Upland rice genotypes evaluation for phosphorus use efficiency. Journal of Plant Nutrition, v.20, p.499-509, 1997. DOI: 10.1080/01904169709365334.

FOULKES, M.J.; HAWKESFORD, M.J.; BARRACLOUGH, P.B.; HOLDSWORTH, M.J.; KERR, S.; KIGHTLEY, S.; SHEWRY, P.R. Identifying traits to improve the nitrogen economy of wheat: recent advances and future prospects. Field Crops Research, v.114. p.329-342, 2009. DOI: 10.1016/j.fcr.2009.09.005.

FOULKES, M.J.; SYLVESTER-BRADLEY, R.; SCOTT, R.K. Evidence for differences between winter wheat cultivars in acquisition of soil mineral nitrogen and uptake and utilization of applied fertilizer nitrogen. The Journal of Agricultural Science, v.130, p.29-44, 1998. DOI: 10.1017/S0021859697005029.

FOULKES, M.J.; SYLVESTER-BRADLEY, R.; WORLAND, A.J.; SNAPE, J.W. Effects of a photoperiod sensitivity gene Ppd-D1 on yield potential and drought resistance in UK winter wheat. Euphytica, v.135, p.63-73, 2004. DOI: 10.1023/B:EU PH.0000009542.06773.13.

GAJU, O.; ALLARD, V.; MARTRE, P.; LE GOUIS, J.; MOREAU, D.; BOGARD, M.; HUBBART, S.; FOULKES, M.J. Nitrogen partitioning and remobilization in relation to leaf senescence, grain yield and grain nitrogen concentration in wheat cultivars. Field Crops Research, v.155, p.213-223, 2014. DOI: 10.1016/j. fcr.2013.09.003.

GAJU, O.; ALLARD, V.; MARTRE, P.; SNAPE, J.W.; HEUMEZ, E.; LEGOUIS, J.; MOREAU, D.; BOGARD, M.; GRIFFITHS, S.; ORFORD, S.; HUBBART, S.; FOULKES, M.J. Identification of traits to improve the nitrogen-use efficiency of wheat genotypes. Field Crops Research, v.123, p.139-152, 2011. DOI: 10.1016/j. fcr.2011.05.010.

GÓRNY,A.G.; BANASZAK, Z.; LUGOWSKA, B.; RATAJCZAK, $D$. Inheritance of the efficiency of nitrogen uptake and utilization in winter wheat (Triticum aestivum L.) under diverse nutrition levels. Euphytica, v.177, p.191-206, 2011. DOI: 10.1007/ s10681-010-0230-z.

GÓRNY, A.G.; GARCZYNSKI, S.; BANASZAK, Z; ŁUGOWSKA, B. Genetic variation in the efficiency of nitrogen utilization and photosynthetic activity of flag leaves among the old and modern germplasm of winter wheat. Journal of Applied Genetics, v.47, p.231-237, 2006. DOI: 10.1007/BF03194628.

GUARDA, G.; PADOVAN, S.; DELOGU, G. Grain yield, nitrogen-use efficiency and baking quality of old and modern Italian bread-wheat cultivars grown at different nitrogen levels. European Journal of Agronomy, v.21, p.181-192, 2004. DOI: 10.1016/j.eja.2003.08.001.

HABASH, D.Z.; BERNARD, S.; SCHONDELMAIER, J.; WEYEN, J.; QUARRIE, S.A. The genetics of nitrogen use in 
hexaploid wheat: $\mathrm{N}$ utilisation, development and yield. Theoretical and Applied Genetics, v.114, p.403-419, 2007. DOI: 10.1007/ s00122-006-0429-5.

HIREL, B.; BERTIN, P.; QUILLERÉ, I.; BOURDONCLE, W.; ATTAGNANT, C.; DELLAY, C.; GOUY, A.; CADIOU, S.; RETAILLIAU, C.; FALQUE, M.; GALLAIS, A. Towards a better understanding of the genetic and physiological basis for nitrogen use efficiency in maize. Plant Physiology, v.125, p.1258-1270, 2001. DOI: 10.1104/pp.125.3.1258.

KING, J.; GAY, A.; SYLVESTER-BRADLEY, R.; BINGHAM, I.; FOULKES, J.; GREGORY, P.; ROBINSON, R. Modelling cereal root systems for water and nitrogen capture: towards an economic optimum. Annals of Botany, v.91, p.383-390, 2003. DOI: 10.1093/ $\mathrm{aob} / \mathrm{mcg} 033$.

LE GOUIS, J.; BÉGHIN, D.; HEUMEZ, E.; PLUCHARD, P. Genetic differences for nitrogen uptake and nitrogen utilisation efficiencies in winter wheat. European Journal of Agronomy, v.12, p.163-173, 2000. DOI: 10.1016/S1161-0301(00)00045-9.

LE GOUIS, J.; GAJU, O.; HUBBART, S.; ALLARD, V.; ORFORD, S.; HEUMEZ, E.; BOGARD, M.; GRIFFITHS, S.; WINGEN, L.; SEMENOV, M.; MARTRE, P.; SNAPE, J.; FOULKES, J. Genetic improvement for an increased nitrogen use efficiency in wheat. Aspects of Applied Biology, v.105, p.151-158, 2010.

MA, B.-L.; WU, T.-Y.; TREMBLAY, N.; DEEN, W.; MCLAUGHLIN, N.B.; MORRISON, M.J.; STEWART, G. On-farm assessment of the amount and timing of nitrogen fertilizer on ammonia volatilization. Agronomy Journal, v.102, p.134-144, 2010. DOI: 10.2134/agronj2009.0021.

MASCLAUX-DAUBRESSE， C.; DANIEL-VEDELE， F.; DECHORGNAT, J.; CHARDON, F.; GAUFICHON, L.; SUZUKI, A. Nitrogen uptake, assimilation and remobilization in plants: challenges for sustainable and productive agriculture. Annals of Botany, v.105, p.1141-1157, 2010. DOI: 10.1093/aob/mcq028.

MOLL, R.H.; KAMPRATH, E.J.; JACKSON, W.A. Analysis and interpretation of factors which contribute to efficiency of nitrogen utilization. Agronomy Journal, v.74, p.562-564, 1982. DOI: 10.2134/agronj1982.00021962007400030037x.

MONTGOMERY, D.C.; PECK, E.A. Introduction to linear regression analysis. New York: John Wiley, 1981. 504p.

ORTIZ-MONASTERIO, J.I.; SAYRE, K.D.; RAJARAM, S.; MCMAHON, M. Genetic progress in wheat yield and nitrogen use efficiency under four nitrogen rates. Crop Science, v.37, p.898-904, 1997. DOI: 10.2135/cropsci1997.0011183X00370003 $0033 \mathrm{x}$.

RAHIMIZADEH, M.; KASHANI, A.; ZARE-FEIZABADI, A.; KOOCHEKI, A.R.; NASSIRI-MAHALLATI, M. Nitrogen use efficiency of wheat as affected by preceding crop, application rate of nitrogen and crop residues. Australian Journal of Crop Science, v.4, p.363-368, 2010.

RESENDE, M.D.V. de; DUARTE, J.B. Precisão e controle de qualidade em experimentos de avaliação de cultivares. Pesquisa Agropecuária Tropical, v.37, p.182-194, 2007.

REYNOLDS, M.P.; RAJARAM, S.; SAYRE, K.D. Physiological and genetic changes of irrigated wheat in the post-green revolution and approaches for meeting projected global demand. Crop Science, v.39, p.1611-1621, 1999. DOI: 10.2135/cropsci1999.3961611x.

RILEY, W.J.; ORTIZ-MONASTERIO, I.; MATSON, P.A. Nitrogen leaching and soil nitrate, nitrite, and ammonium levels under irrigated wheat in Northern Mexico. Nutrient Cycling in Agroecosystems, v.61, p.223-236, 2001. DOI: 10.1023/A:1013758116346.

SADRAS, V.O.; LAWSON, C. Nitrogen and water-use efficiency of Australian wheat varieties released between 1958 and 2007. European Journal of Agronomy, v.46, p.34-41, 2013. DOI: 10.1016/j.eja.2012.11.008.

SYLVESTER-BRADLEY， R.; KINDRED， D.R. Analysing nitrogen responses of cereals to prioritize routes to the improvement of nitrogen use efficiency. Journal of Experimental Botany, v.60, p.1939-1951, 2009. DOI: 10.1093/jxb/erp116.

TEDESCO, M.J.; GIANELLO, C.; BISSANI, C.A.; BOHNEN, H.; VOLKWEISS, S.J. Análise de solo, plantas e outros materiais. 2.ed. Porto Alegre: Universidade Federal de Rio Grande do Sul, 1995. 174p.

WRIGHT, S. Correlation and causation. Journal of Agricultural Research, v.20, p.557-585, 1921.

XU, G.; FAN, X.; MILLER, A.J. Plant nitrogen assimilation and use efficiency. Annual Review of Plant Biology, v.63, p.153-182, 2012. DOI: 10.1146/annurev-arplant-042811-105532.

ZHAO, R.-F.; CHEN, X.-P.; ZHANG, F.-S.; ZHANG, H.; SCHRODER, J.; ROMHELD, V. Fertilization and nitrogen balance in a wheat-maize rotation system in North China. Agronomy Journal, v.98, p.935-945, 2006. DOI: 10.2134/agronj2005.0157.

Recebido em 30 de maio de 2014 e aprovado em 10 de setembro de 2014 